\title{
Process sustainability prediction: a computer aided design tool for sustainable industrial development
}

\author{
M. Fermeglia, L. Toma \& G. Longo \\ Department of Industrial Engineering \& Information Technology, \\ University of Trieste, Italy
}

\begin{abstract}
Chemical process sustainability can be estimated using different sustainability indicators. The quantitative estimation of those indicators is necessary (i) for evaluating the environmental impact of a chemical process and (ii) for choosing the best design among different available alternatives. To accomplish these goals, the computerized calculation of sustainability indicators requires the use of at least three computer tools: (i) process simulation, (ii) molecular modelling and a (iii) sustainability indicators software code. In this paper, a complete software platform, Process Sustainability Prediction Framework, integrated with process simulation programs, which support the CAPE-OPEN interfaces, is presented, discussed and applied to an example of biofuel production. The process considered is implemented in a real plant in Cuba.
\end{abstract}

Keywords: sustainability, process design, material impact, energy impact, Cape Open, process simulation, environmental impact, PSP, biofuels, bioethanol.

\section{Introduction}

Sustainable development has been defined as the creation of goods and services using processes and systems that are non-polluting; conserving energy and natural resources; economically viable; safe and healthy for employees, communities and consumers; socially and creatively rewarding for all working people (Veleva and Ellenbecker [1]).

In the sustainable development context, an important role is played by chemical engineering, basically for two main reasons: 1) the production of 
chemical process plants contributes greatly to national income and is absolutely essential to modern society; thus, the society development depends on the development of the chemical process industry and vice versa; 2) many critical environmental problems can be associated directly with chemical processes or to the use of chemical products and by-products that result in waste streams to soil, water and air (Korevaar [2]). Chemical industry produces materials for multiple consumer markets, which need to be produced, used and recycled by manufacturing processes that are clean, safe and economical (Jenck et al. [3]).

In order to eliminate or reduce negative environmental problems, the environmental performance of chemical processes should be identified and quantified at an early stage of process design. Taking into account the environmental aspects at design time, an answer to "which design is more environmentally friendly, and implicitly more sustainable, for a specific chemical process?" can be provided.

The present paper is concerned with the description and application of a specific software tool, Process Sustainability Prediction (PSP) Framework.

There are several advantages deriving from the use of PSP. The most important advantage is the possibility of including the potential environmental impact criteria in the optimization of the process, on top of the traditional technical and economic criteria. Modern tools such as process simulation may result in several benefits for the design of a chemical process. These benefits include but are not limited to (i) shortened product-process development cycles, (ii) optimization of existing processes to improve energy efficiency and minimize production of waste, (iii) efficient design of new products and processes and (iv) improvements in health, safety, and environment (Fermeglia et al. [4]).

\section{The evaluation of the sustainability of a process}

Economy, society and environment are three basic elements of sustainability. Each of these elements is connected to, and dependent upon, the others (NJSSI [5]). It is generally acknowledged that sustainability results from a balance among the three aspects. The selection of an adequate set of indicators for sustainability evaluation is very important, for comparative analysis between different versions of a process (Sikdar [6]). With the objective of providing an easy-to-implement method for applying indicators for the purpose of analysing industrial systems for sustainability, Sikdar proposed a typology of indicators, considering the three dimensions of sustainability in three distinct hierarchical groups: (1) One dimensional (1D) indicators, which provide information about only one dimension of sustainability: economical, ecological, or social; (2) Two dimensional (2D) indicators, which provide information simultaneously about two dimensions of sustainability: socio-ecological, socio-economical, or economic-ecological; (3) Three dimensional (3D) indicators, which provide information about all three dimensions of sustainability (Martins et al. [7]).

Fermeglia et al. [8] proposed to analyse four 3D and four 1D indicators as representative for the $3 \mathrm{D}$ and $1 \mathrm{D}$ analysis respectively. The reason why the 
attention is focused to 3D and 1D analysis is that the former is sufficiently general and not very complicate to carry out at design time of a process and considers all possible aspects of the sustainability, while the latter is very precise, technically oriented and directly applicable to engineering practice. In the following paragraphs a brief introduction to the method is given (Fermeglia et al. [8]).

\subsection{Three dimensional indicators}

The Material Intensity (MI) Indicator measures the amount of non-renewable resources required to obtain a unit mass of products. It includes raw materials, solvents, and other ingredients (Martins et al. [7]). Details regarding the calculation of this indicator are given by Schwarz et al. [9]. The Energy Intensity (EI) Indicator measures the energy demands of the process. It is calculated per unit mass of products and focuses primarily on the use of non-renewable energy (Martins et al. [7]). Details about the calculation of this indicator are given by Tanzil et al. [10]. The Potential Chemical Risk Evaluation (PCRE) Indicator regards the risk associated with the manipulation, storage and use of hazardous chemical substances in the process. It is schematically represented in Figure 1.

The chemicals used in the process under study are classified depending on their relative quantity, frequency of use, danger characteristics and potential exposure. Thus, for each chemical, the designated "frequency class", "quantity class", "hazard class" and "potential exposure class" are determined according to Vincent (Vincent et al. [11]).

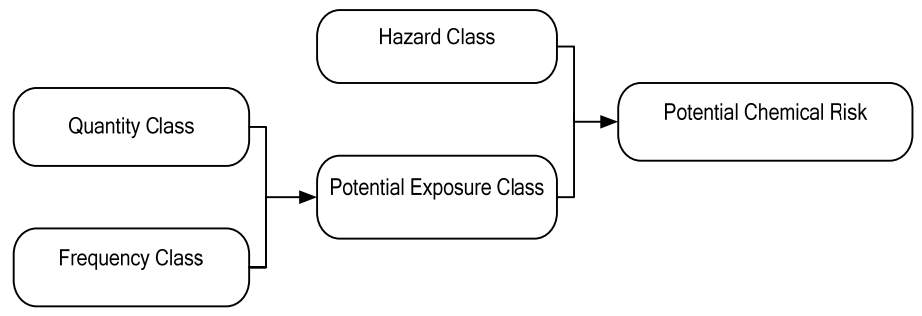

Figure 1: Potential chemical risk evaluation.

The Potential Environmental Impact Indicator is due to the emissions and discharge of hazardous chemicals to the environment. It is similar to the one for the potential chemical risk, as represented in Figure 2.

The chemicals are classified according to their relative quantity and hazard characteristics. Furthermore, the potential environmental impact depends on the transfer to a receiver medium, such as air, water or soil, which is governed by physicochemical characteristics and transfer coefficients as shown in Figure 2 (Martins et al. [7]). 


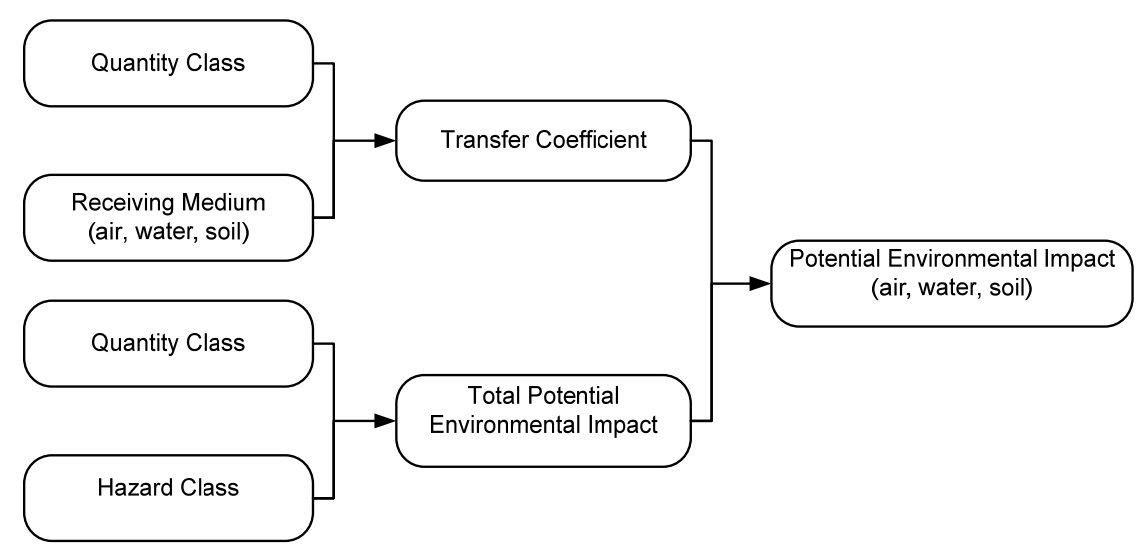

Figure 2: $\quad$ Potential environmental impact evaluation.

\subsection{One dimensional indicator - the Waste Reduction (WAR) Algorithm}

The WAR Algorithm, based on the concept of environmental impact balance, analogous to mass or energy balances, uses data from the process (stream flowrates and mass fractions) and toxicological data to calculate the environmental impact of a chemical plant (Cabezas et al. [12]; Young and Cabezas [13]; Young et al. [14]).

Toxicological data can be grouped in different environmental impact categories: Global Warming Potential (GWP), Acidification Potential (AP), Ozone Depletion Potential (ODP), Photochemical Oxidation or Smog Formation Potential (PCOP), Human Toxicity Potential by Ingestion (HTPI), Human Toxicity Potential by Inhalation or Dermal Exposure (HTPE), Aquatic Toxicity Potential (ATP), Terrestrial Toxicity Potential (TTP). They are used in evaluation of potential environmental impact using the WAR [13].

GWP is determined by comparing the extent to which a unit mass of a chemical absorbs infrared radiation over its atmospheric lifetime to the extent the $\mathrm{CO}_{2}$ absorbs infrared radiation over its respective lifetimes. AP or acid rain potential is determined by comparing the rate of release of $\mathrm{H}+$ in the atmosphere as promoted by a chemical to the rate of release of $\mathrm{H}+$ in the atmosphere as promoted by $\mathrm{SO}_{2}$. ODP is determined by comparing the rate at which a unit mass of chemical reacts with ozone to form molecular oxygen to the rate at which a unit mass of CFC-11 (trichlorofluoromethane) reacts with ozone to form molecular oxygen. PCOP or smog formation potential is determined by comparing the rate at which a unit mass of chemical reacts with a hydroxyl radical $\mathrm{OH}$ to the rate at which a unit mass of ethylene reacts with $\mathrm{OH}$. HTPI is calculated for a chemical if it existed as a liquid or solid at a temperature of $0^{\circ} \mathrm{C}$ and atmospheric pressure. As a first approximation, the lethal-dose that produced death in $50 \%$ of rats by oral ingestion (LD50) has been used as an estimate for the HTPI. HTPE was determined for that chemical if it existed as a gas a temperature of $0^{\circ} \mathrm{C}$ and atmospheric pressure. ATP was estimated by using toxicological data for a single, representative species of fish, Pimephales 
promelas (Fathead Minnows). The data for this assay comes in the form of a LC50, a lethal concentration which causes death in $50 \%$ of the test specimens. Different molecular modeling methods to evaluate the thermo-physical properties which lead to environmental impact categories are used in the PSP framework [8].

The four 1D environmental impact indexes used in WAR Algorithm and in the PSP Framework are: the total rate of impact output, the total impact output per mass of products, the total rate of impact generation and the total impact generated per mass of product. Those indexes are referred in the PSP Framework as Iout, Iout mp, Igen and Igen_mp. The analysis of such indexes permits to select the most environmentally friendly design among different process alternatives.

\section{The Process Sustainability Prediction (PSP) framework}

The PSP Framework implementation has been made according to Martins and Vincent for 3D Indicators (Martins et al. [7]; Vincent et al. [11]) and to Young and Cabezas for 1D Indicators (Cabezas et al. [12]; Young and Cabezas [13]; Young et al. [14]). A general schema illustrating the main steps of the development is given in Figure 3.

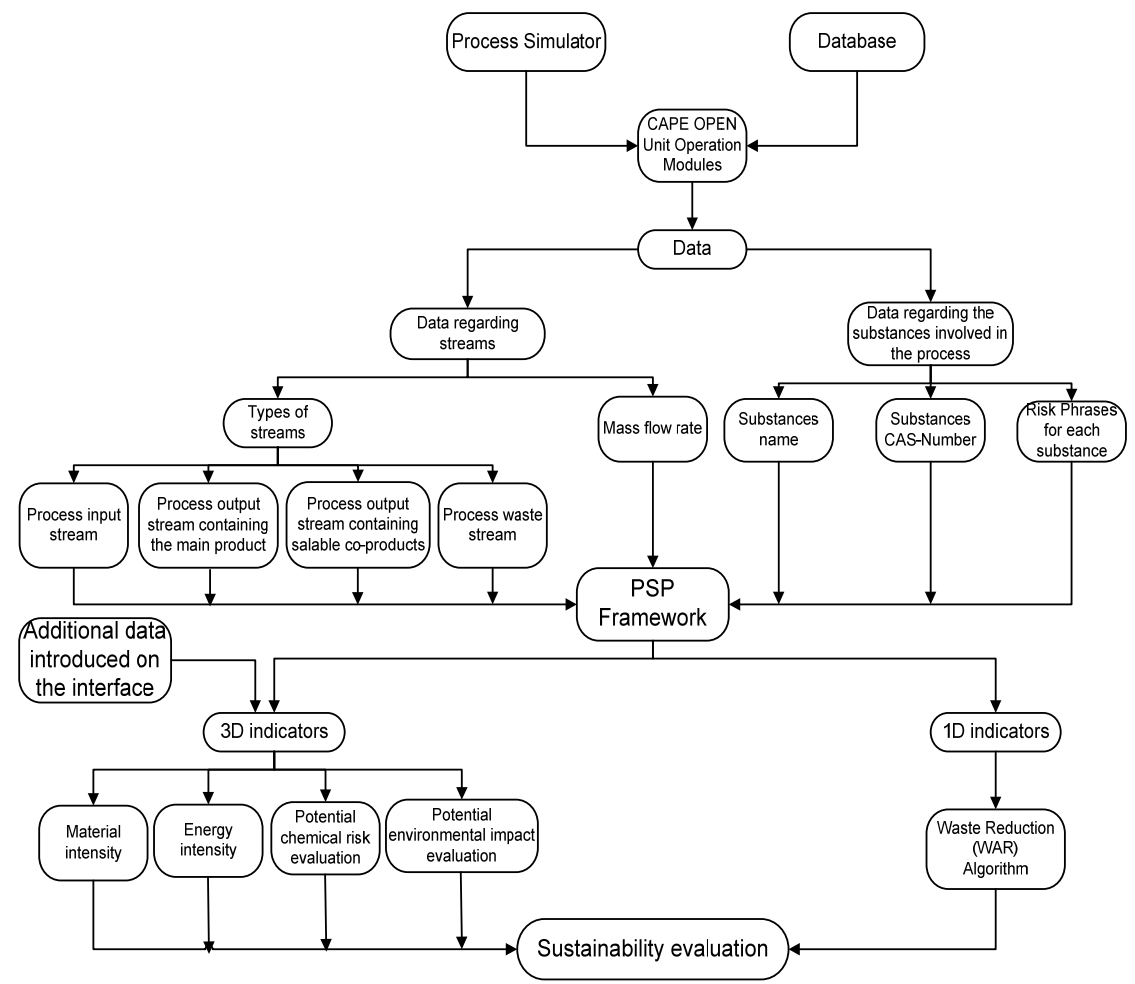

Figure 3: General schema of PSP framework development. 
The two starting points for calculating the sustainability indicators are the process simulator and the database containing toxicity and risk phrases data. In a previous paper [8] we have developed methods for the estimation of toxicological properties using molecular modeling techniques. Using these methods, as well as resorting to experimental toxicological data, allowed us to generate a data base that can be used in PSP. The other issue, namely the interface to the process simulators, is addressed by developing CAPE OPEN compliant modules for accessing the data in the simulators.

The CAPE OPEN technology has been chosen since it is the only available inter platform standard in the process simulation sector. Four CAPE OPEN (CO) Modules have been developed (two CO Modules for 3D Indicators and two CO Modules for 1D Indicators).

These modules are inserted in the process flow-sheet, as any other unit operation, with the aim of extracting all the necessary data requested for further calculations (flow-rate quantities, flow-rate composition, energy demand of the process, toxicological data, risk phrases data). The CO modules in PSP are directly connected to the toxicological database (DB), containing all the necessary data for the evaluation of the indexes for about 4900 compounds.

The data obtained through the $\mathrm{CO}$ Modules are processed in the PSP software. Other information such as $\mathrm{H}_{2} \mathrm{O}, \mathrm{O}_{2}, \mathrm{~N}_{2}$ moles formed in the reaction; energy demand of the process; frequency of use and physical state of each substance involved are supplied by the user in the PSP interfaces. Details regarding the implementation of the $\mathrm{CO}$ modules, the development of the toxicological database, the PSP software and its applications are given by Toma [15].

\section{PSP framework applications}

Application of the PSP framework has been done in different contexts. Traditional chemical processes as well as innovative processes in the area of energy generation, environment protection, food processing and biotechnology have been analyzed (Toma [15], Fermeglia et al. [8]). The PSP framework, being Cape Open compliant, can be easily and directly used in any process simulator: it has been tested with PROII (Avensys-SimSci), Aspen+ (Aspentech) and CocoCofe (AmsterChem). In this paper we present an example of application of the PSP framework to a bioethanol production plant from sugar cane molasses.

\subsection{Bioethanol production process: process simulation}

Molasses differs from other feed stocks for alcohol production such as corn, millo and potatoes in that these plant products contain carbohydrate stored as starch. Indeed, these feedstocks must be pre-treated by enzymatic action to hydrolyze starch into fermentable sugars. In contrast, the carbohydrates in molasses are already in the form of sugars and need no pretreatment. The simplest form of sugar is glucose, which is fermented by normal distillery and brewery yeasts. Sucrose is the principal sugar contained in molasses and is 
readily fermentable either directly, or as its glucose and fructose components. Degradation with added water is referred to as hydrolysis. This addition of water should be taken into consideration when making any calculations of the amount of simple sugars (monosaccharides) produced from disaccharides.

In the production of cane sugar, the cane is crushed in a mill to squeeze out the juice. The juice is heated, clarified by filtration and the addition of lime (to remove cane fibers and sludge) and then evaporated to concentrate the sugar and crystallized. The syrup containing the crystals is then centrifuged to separate the crystals and the syrup residue (which still has a high content of sugar). The residue is referred to as "A molasses". It is evaporated and centrifuged again to recover more crystalline sugar; and the syrup residue is now referred to as " $\mathrm{B}$ molasses". The process may be repeated to yield more sugar and a "C molasses" as residue. Sugar mills normally evaporate and centrifuge a maximum of three times, but the number of treatments will depend on marketplace economics [16]. In addition to ethanol, yeast also produces other substances such as glycerol and succinic acid. Many distillers, therefore, use $95 \%$ of the $51.11 \% \mathrm{w} / \mathrm{w}$ figure as a realistic basis for calculating expected ethanol yields. For costing purposes, one can expect to obtain about 219.6 liters of ethanol from a ton of molasses at $46 \%$ sugars (TSAI).

Molasses fermentations may be conducted at $30-36^{\circ} \mathrm{C}$, but if one is aiming for high final alcohol levels in the beer it is advisable to lower the temperature.

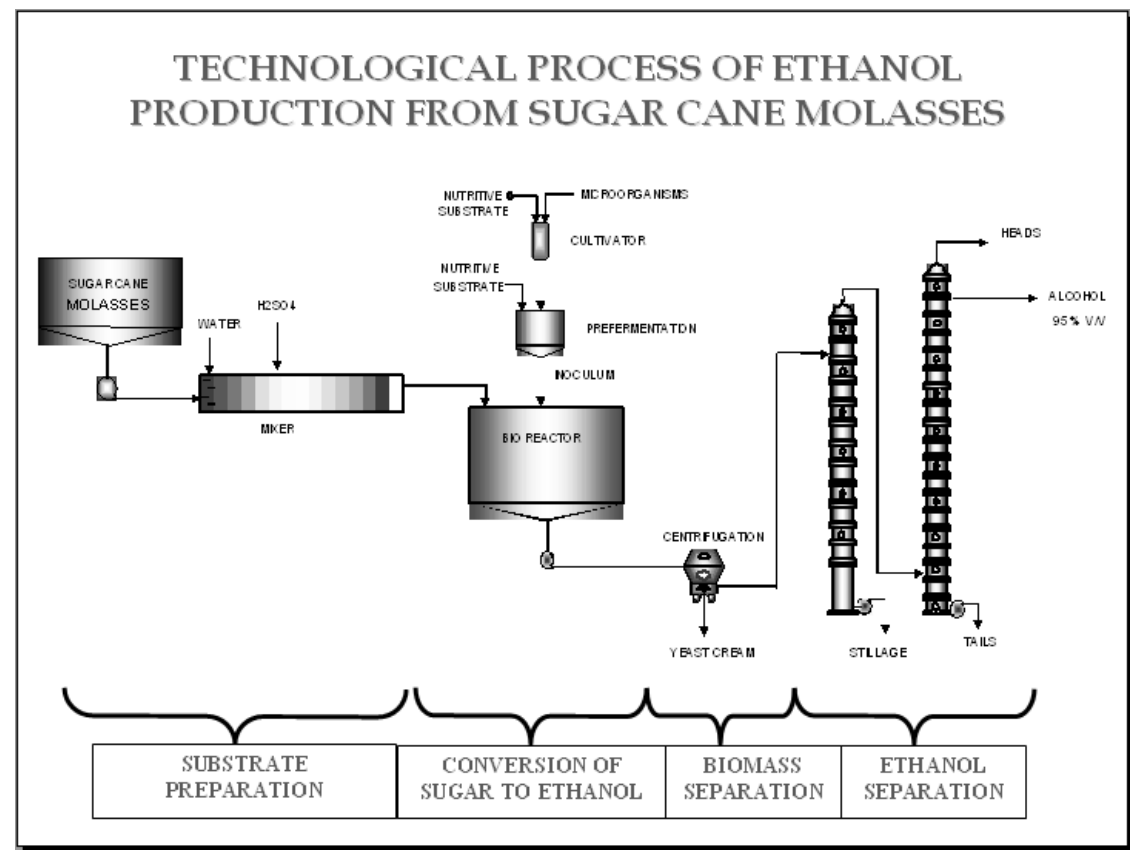

Figure 4: Bioethanol production from sugar cane molasses. 
Molasses fermentations are generally much more rapid than grain fermentations and can be completed in half the time required for grain. This means that much more heat is produced per hour, which necessitates better cooling facilities than for grain fermentations. Some alcohol plants share one external heat exchanger between two fermenters.

The main problem in distillation of molasses is scale formation in the stripping column. A second problem, foaming in the distillation columns, is encountered with molasses. Foaming frequently limits the rate of distillation; and if uncontrolled it may pass from the stripping column into the rectifier and cause problems of proof, color, etc. Foaming is best controlled by continuous injection of a suitable antifoam into the mash feed line as this ensures that all the mash reaching the column is adequately dosed.

The Distillery "Paraiso", located in the central region of Cuba was selected for the simulation. The process is schematically illustrated in figure 4 . The process is divided into the following steps: (i) Substrate Preparation, (ii) Conversion Sugar to ethanol (Yeast propagation and Alcoholic fermentation stage), (iii) Biomass Separation, (iv) Ethanol separation (Striping and Rectification section) [16].

The distillery has a capacity of 700001 of hydrated grade alcohol per day [16].

\subsection{Bioethanol production process: sustainability analysis}

The flow sheet of the simulation is shown in Figure 5. The diagram includes the substrate preparation, conversion sugar to ethanol (Yeast propagation and Alcoholic fermentation stage), biomass separation and ethanol separation (Striping and Rectification section).

Sensitivity studies for the process of interest have shown that the quantity of ethanol produced is sensitive to the temperature of the fermentation reactor, as anticipated in the description of the process [16]. For this reason the first case simulated corresponds to a temperature of the fermentation reactor of $46^{\circ} \mathrm{C}$. In the second case the temperature of the fermentation reactor is lowered to $34^{\circ} \mathrm{C}$. These two cases are examples of modification of the process conditions with no changes in the equipment schema. In the third case, the washing (final) column is not present, thus representing an example of process modification. The 3D indicators obtained using the PSP Framework are reported in Table 1.

CASE 1 shows the lowest MI and EI indicators. All three processes are continuous processes, so the frequency class for the substances present in the process is the same (permanent use; $>6 \mathrm{~h} /$ day) as well as the risk phrases, since there is no difference for the components involved in the process.

PEI has the same values for all three cases because the physical states of the substances involved in the process are the same and the receiving medium (air, water, soil) is also the same.

Therefore, due to the more favourable values of MI and EI, CASE 1 represents the best process condition in term of environmental impact among the cases considered. 


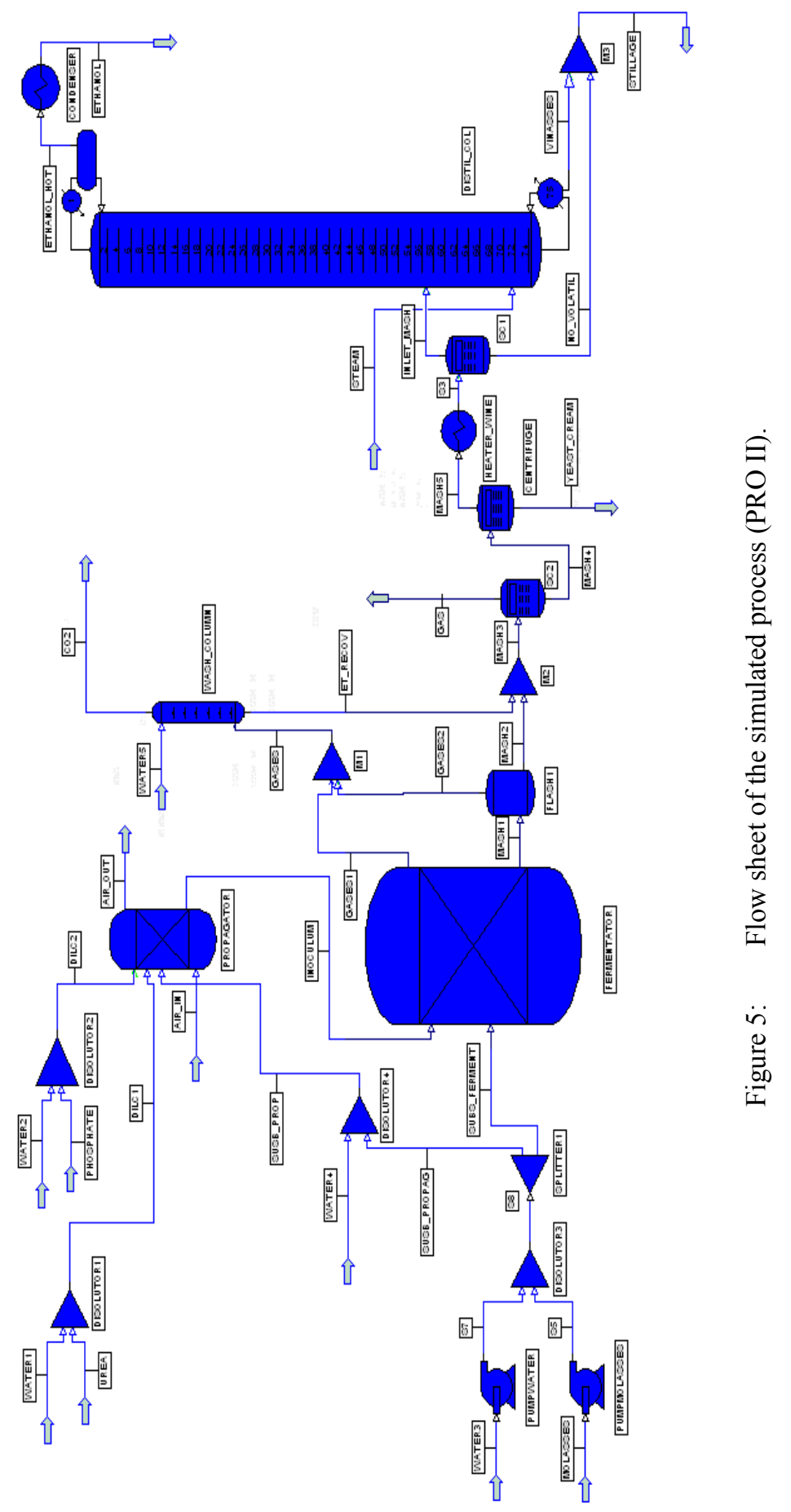


For the same cases, WAR Algorithm, namely the 1D analysis, has been applied. The results obtained using 1D indicator, are reported in Table 2.

Table 1: $\quad 3 \mathrm{D}$ indicators for the different cases investigated.

\begin{tabular}{lcccc}
\hline \multirow{2}{*}{ CASE STUDY } & \multicolumn{4}{c}{ 3D INDICATORS } \\
\cline { 2 - 5 } & MI & EI & PCR & PEI \\
\hline CASE 1 & 53125.1357 & 2.0566 & 355 & 8642.38 \\
\hline CASE 2 & 53129.9006 & 2.7069 & 355 & 8642.38 \\
\hline CASE 3 & 53364.8938 & 2.2137 & 355 & 8642.38 \\
\hline
\end{tabular}

Table 2: $\quad$ 1D indicator (WAR algorithm) for the different cases investigated.

\begin{tabular}{lcccc}
\hline CASE & \multicolumn{4}{c}{ ENIVORONMENTAL INDEXES NAME } \\
\cline { 2 - 5 } STUDY & $\begin{array}{c}\text { Iout } \\
(\mathrm{PEI} / \mathrm{hr})\end{array}$ & $\begin{array}{c}\text { Iout_mp(P } \\
\mathrm{EI} / \mathrm{kg})\end{array}$ & $\begin{array}{c}\text { Igen } \\
(\mathrm{PEI} / \mathrm{hr})\end{array}$ & $\begin{array}{c}\text { Igen_mp } \\
(\mathrm{PEI} / \mathrm{kg})\end{array}$ \\
\hline CASE 1 & 3100.47438 & 1.7722 & -54.4912 & $-3.11 \mathrm{E}-02$ \\
\hline CASE 2 & 3107.46802 & 1.7594 & -47.4976 & $-2.69 \mathrm{E}-02$ \\
\hline CASE 3 & 3123.40115 & 1.7902 & -31.5645 & $-1.81 \mathrm{E}-02$ \\
\hline
\end{tabular}

CASE 3 results to have a high impact for the 3D and the $1 \mathrm{D}$ analysis. This is due to the absence of the scrubbing tower, and the consequent higher amount of gases emitted to the atmosphere In CASE 1 and 2 gases are recovered, thus lowering the impact of the process.

CASE 1 and 2 do not differ a lot, since the equipment are the same and the only difference is the reaction condition: CASE 2 needs a higher amount of heat at the reboiler, this justifying a higher energy consumption.

In summary, CASE 1 is the most favorable in terms of environmental impact.

\section{Conclusions}

A methodology to quantify the environmental impact of a chemical process is presented, discussed and applied to a process for the production of biofuels. The methodology, used in the early stages of project design, allows choosing the most environmentally friendly design among different process alternatives.

The software presented, the PSP Framework, contains the following items: (i) CAPE OPEN Modules ready to be used in any process simulator which provides $\mathrm{CO}$ Interfaces, (ii) a toxicological database, and (iii) various support program codes developed in Visual Basic.

The connection between the process simulator and the environmental evaluation tools has been done using a standard technique: CAPE OPEN. Four $\mathrm{CO}$ Modules, with different characteristics and aims have been developed in order to get automatically the necessary information from the process simulator. The modules have been tested successfully using different process simulators such as: PROII, Aspen Plus, COCO/COFE. 
A relational database containing 4900 substances has been created and implemented and it is available in the PSP framework.

The ethanol production process from sugar cane molasses has been simulated using PROII process simulator. Three process alternatives are proposed and analyzed in order to choose the most environmentally friendly one.

The most environmentally friendly design is the first one, as it can be noticed from the results obtained using the 3D and 1D indicators of the PSP Framework.

In conclusion, the PSP framework proved to be a very useful decision support tool in the design phase of a process. Several different process alternatives, in terms of process conditions, process feeds and operative settings may be easily compared in terms of environmental impact. Using PSP it is possible to identify the best scenario that reduces the impact to the environment by using only in silico' calculations. The PSP application may lead to a new era of optimization in the process design industry and could be applied in developed, developing and emerging countries for a complete support to the global sustainability.

\section{References}

[1] Veleva V, Ellenbecker M. Indicators of sustainable production: framework and methodology. Journal of Cleaner Production. 2001; 9:519-549.

[2] Korevaar G. Sustainable Chemical Processes and Products- New Design Methodology and Design Tools. 2004. PhD Thesis- Technical University of Delft.

[3] Jenck JF, Agterberg F, Droescher M. Products and processes for a sustainable chemical indutry: a review of achievements and prospects. Green Chemistry.2004; 6: 544-556.

[4] Fermeglia M, Pricl S, Longo G. Molecular Modelling and Process Simulation: Real Possibilities and Challenges. Chemical and Biochemical Engineering Quarterly. 2003; 17: 69-79.

[5] "NJSSI" (2007). Available from: New Jersey Sustainable State Institute $<$ http://njssi.org/about.asp?Level2ItemID $=55>\quad$ (last accessed October 2007)

[6] Sikdar SK. Sustainable Development and Sustainable Metrics. AIChE Journal. 2003; 49: 1928-1932.

[7] Martins AA., Mata TM, Costa CAV, Sikdar SK. Framework for Sustainability Metrics, Industrial and Engineering Chemical Research. 2007; 46: 2962 -2973.

[8] Fermeglia M., Longo G., Toma L., Computer aided design for sustainable industrial processes: specific tools and applications, AIChE Journal, 55: 1065-1078 (2009).

[9] Schwarz J, Beloff B, Beaver E. Use Sustainability Metrics to Guide Decision-Making. Environmental Protection. Available from: $<$ http://www.bridgestos.org/CEP-July.pdf $>$ (last accessed October 2007).

[10] Tanzil D, Ma G, Beloff B. Automating the Sustainability Metrics Approach. AIChESpring Meeting, 25-29 April 2004, New Orleans. Available from: BRIDGES to Sustainability Institute 
$<$ http://www.bridgestos.org/AIChESpring2004MetricsPPT.pdf $>\quad$ (last accessed October 2007).

[11] Vincent R, Bonthoux F, Mallet G, Iparraguirre JF, Rio S. Méthodologie d'Evalution Simplifiée du Risque Chimique: Un Outil d'Aide à la Décision. INRS Hyg. Secur. Travail. 2005; 195 .

[12] Cabezas H, Bare JC, Mallick SK. Pollution Prevention with Chemical Process Simulators: The Generalized Waste Reduction (WAR) Algorithm. Computers \& Chemical Engineering. 1997; 21: 305-310.

[13] Young DM, Cabezas H. Designing Sustainable processes with simulation: the waste reduction (WAR) algorithm. Computers and Chemical Engineering. 1999; 23: 1477-1491.

[14] Young D, Scharp R, Cabezas H. The waste reduction (WAR) algorithm: environmental impacts, energy consumption, and engineering economics. Waste Management. 2000; 20: 605-615.

[15] Toma L. Computer Aided Design of Sustainable Industrial Processes. 2008. PhD Thesis- University of Padua, Italy, 2008.

[16] Domenech López F., Bioethanol production from sugar cane molassa: process simualtion and optimization, Internal report, ICS-UNIDO, Trieste, November 2005. 